\title{
NGHIÊN CÚU CÁC YẾU TỐ ẢNH HƯởNG ĐẾN Ý ĐỊNH SỬ DỤNG ÚNG DỤNG GIAO THỨC ĂN CỦA NGƯỜI TIỂU DÙNG TẠI THÀNH PHỐ ĐÀ NÃ̃NG
}

\author{
LÊ NAM HẢI, PHAN THI TRÚC MAI \\ Trường Đại học Công nghiệp thành phố Hồ Chi Minh \\ lenamhai@iuh.edu.vn
}

Tóm tắt: Nghiên cứu được thực hiện nhằm mục đích xem xét các yếu tố tác động đến ý định sử dụng ứng dụng giao thức ăn của người tiêu dùng. Dữ liệu phân tích dựa trên 326 bảng khảo sát hợp lệ được thu thập từ người tiêu dùng tại khu vực $\mathrm{TP}$. Đà Nẵng. Các phương pháp phân tích chính được sử dụng bao gồm: phân tích độ tin cậy của thang đo (Cronbach's Alpha), phân tích nhân tố khám phá (EFA) và phân tích hồi quy đa biến. Kết quả nghiên cứu cho thấy có năm yếu tố: Nhận thức tính dễ sử dụng, mong đợi về giá, nhận thức sự thuận tiện, niềm tin và chuẩn chủ quan thực sự có ảnh hưởng tích cực đến ý định sử dụng. Bên cạnh đó, nghiên cứu cũng đề xuất hàm ý quản trị nhằm giúp các doanh nghiệp về ứng dụng giao thức ăn có thể nâng cao chất lượng, đề ra hướng phát triển và mở rộng thị trường

Từ khóa: Chấp nhận công nghệ, hành vi người tiêu dùng, thương mại di động, ứng dụng giao thức ăn, ý định sử dụng.

\section{A STUDY OF FACTORS INFLUENCING THE INTENTION TO USE FOOD DELIVERY APPLICATIONS OF CONSUMERS IN DA NANG CITY}

\begin{abstract}
The purpose of this research is to examine factors affecting the intention to use food delivery applications of consumers. The study analyzed data of 326 suitable questionnaires collected from consumers in Da Nang City. The main methods used in this research include: the Cronbach's Alpha test of reliability, exploratory factor analysis (EFA), and multiple regression analysis. The findings show five factors such as perceived easy to use, perceived price, perceived convenience, trust and subjective norm having positive impacts on consumers' intention to use food delivery applications. In addition, this study has also proposed some managerial implications for food delivery apps supplier to improve quality, to set the direction of development and to expand market.
\end{abstract}

Keywords: technology acceptance, consumer behavior, mobile commerce, food delivery application, intention to use.

\section{GIỚI THIỆU}

Thời đại công nghệ lên ngôi, nhịp sống bận rộn đã dẫn đến những thay đổi đáng kể trong thói quen tiêu dùng của nhiều người khi họ dần chuyển từ việc mua sắm trực tiếp sang sử dụng thương mại điện tử. Bên cạnh đó, sự phát triển nhanh chóng của công nghệ thông tin với tần suất sử dụng Internet tăng cao thì các thiết bị di động không còn đơn thuần là một công cụ liên lạc mà dần trở thành một phương tiện cung cấp các tính năng tiện lợi cho người sử dụng như nghe nhạc, xem phim, mua sắm... Và một trong số đó có thể kể đến là ứng dụng giao thức ăn. Năm 2018 vừa qua được xem là một cuộc thay đổi lớn đối với thị trường giao nhận thức ăn trực tuyến Việt Nam khi xu hướng sử dụng ứng dụng giao thức ăn thay cho việc trực tiếp đến các nhà hàng, quán ăn để ăn uống của người tiêu dùng có sự tăng tưởng mạnh. Theo nghiên cứu của GCOMM cuối năm 2018 cho thấy, tần suất sử dụng các dịch vụ đặt món trực tuyến của người thành thị hiện khá cao. Cụ thể, 99\% người tham gia khảo sát cho biết sử dụng các dịch vụ đặt thức ăn trực tuyến ít nhất 2-3 lần/tháng, trong đó 39\% người tham gia khảo sát đặt món thông qua ứng dụng 2-3 lần/tuần [5]. Nắm bắt nhu cầu cũng như thấu hiểu được tâm lý của người tiêu dùng, hàng loạt ứng dụng giao thức ăn như Grabfood, Now.vn, GoViet, Lozi...lần lượt ra đời với phương thức sử dụng đơn giản, tiết kiệm thời gian, giao dịch nhanh chóng và hiệu quả cao hơn đang dần được khách hàng ưa chuộng. Việc đặt hàng trực tuyến đã dần phát triển nhanh chóng, không chỉ dừng lại ở cách thỏa mãn nhu cầu, cung ứng sản phẩm mà 
còn tác động và làm thay đổi đáng kể thói quen tiêu dùng; đồng thời đang tạo ra cuộc đua vô cùng khốc liệt giữa các thương hiệu vài năm trở lại đây.

Tuy có sự chuyển biến lớn đối với thị trường giao nhận thức ăn trực tuyến, nhưng do tính chất mới mẻ, chỉ phát triển mạnh tại một số khu vực như TPHCM, Hà Nội..., cũng như ứng dụng công nghệ không phải lúc nào cũng phù hợp với tất cả các đối tượng khách hàng thì cần phải có sự quan tâm đúng mức đến nhận thức người tiêu dùng, có các hướng triển khai tại những khu vực mới như Đà Nẵng, Nha Trang, Biên Hòa...giúp phát triển thị trường đặt món trực tuyến vẫn còn nhiều tiềm năng và mở rộng phạm vi.

TP. Đà Nẵng được xem là một trong những vùng kinh tế trọng điểm của khu vực Miền Trung - Tây Nguyên khi chỉ trong vòng vài năm trở lại đây, nền kinh tế của Thành phố này liên tục có sự tăng trưởng vượt trội. Tình hình kinh tế - xã hội TP.Đà Nẵng năm 2018 tiếp tục phát triển với 9 chỉ tiêu kinh tể chủ yểu đều tăng trưởng khá, trong đó $6 / 9$ chỉ tiêu đạt và vượt kế hoạch [2]. Đóng góp chính vào mức tăng trưởng cao và ổn định của kinh tế TP. Đà Nẵng chủ yếu từ hai nhóm ngành dịch vụ và công nghiệp - xây dựng. Thành phố có xu hướng chuyển dịch từ nông nghiệp sang công nghiệp - dịch vụ, đồng thời đây cũng là khu vực có hơn 9 ngàn người dân, là cửa ngõ giao thương và tiếp cận công nghệ mới, liên tục có các chương trình giao lưu công nghệ, thương mại di động cũng giúp mở ra cho Đà Nẵng tiềm năng lớn mạnh trong lĩnh vực này. Sự chuyển mình nhanh chóng của $\mathrm{TP}$. Đà Nẵng là một tín hiệu đáng mừng và đây chắc hẳn sẽ là một thị trường tiềm năng trong tương lai giữa cuộc đua giao nhận của các thương hiệu.

Với những lý do trên, nhìn nhận được thực trạng tiềm năng của thị trường, cũng như nhận thấy việc nghiên cứu, tìm ra các yếu tố ảnh hưởng đến ý định sử dụng của người tiêu dung tại TP. Đà Nẳng trong thời điểm hiện tại là hết sức cần thiết cả về mặt học thuật lẫn thực tiễn, nhóm tác giả quyết định triển khai mô hình nghiên cứu các yếu tố ảnh hưởng đến ý định sử dụng ứng dụng giao thức ăn của người tiêu dùng tại TP. Đà Nẵng. Nghiên cứu sẽ giúp bổ sung các lập luận, đồng thời là bằng chứng thực nghiệm góp phần giúp các doanh nghiệp tìm ra hướng phát triển mới, nâng cao giải pháp giúp thu hút khách hàng sử dụng dịch vụ, xây dựng chiến lược tiếp thị và phát triển hình thức giao nhận thức ăn trực tuyến của mình tại thành phố này trong thời gian tới. Bên cạnh phần giới thiệu, các nội dụng còn lại của bài viết sẽ trình bày về: cơ sở lý thuyết và mô hình nghiên cứu; phương pháp nghiên cứu; kết quả nghiên cứu; kết luận và hàm ý quản trị.

\section{CƠ SỞ LÝ THUYẾT VÀ MÔ HÌNH NGHIÊN CỨU}

\section{1 Ứng dụng giao thức ăn}

Trong xu thế hiện nay, cùng với sự tăng trưởng mạnh của công nghệ thông tin thì người tiêu dùng đã dần có xu hướng chuyển sang sử dụng các thiết bị điện tử để phục vụ cho nhu cầu mua sắm, vui chơi giải trí, trò chuyện cùng bạn bè nhiều hơn. Thương mại di động cũng từ đó mà phát triển một cách vượt trội chỉ trong một khoảng thời gian ngắn. Các thiết bị di động không còn đơn thuần là một công cụ để liên lạc, nghe nhạc, xem phim mà dần trở thành một phương tiện hỗ trợ người tiêu dùng trong việc mua sắm, đặt món ăn trực tuyến thông qua việc sử dụng các ứng dụng tiện ích trên điện thoại. Và một trong số đó có thể kể đến là ứng dụng giao thức ăn.

Theo Kimes và ctg (2011) [17] "Đặt món ăn trực tuyến là quá trình đặt hàng thông qua trang web (ứng dụng di động) của nhà hàng hoặc thông qua trang web (ứng dụng) của nhiều nhà hàng. Một khách hàng có thể chọn để giao đồ ăn hoặc nhận. Việc thanh toán cũng được quản lý thông qua ứng dụng (trang web) hoặc bằng tiền mặt tại nhà hàng khi đi lấy hàng." Bên cạnh đó He và ctg (2018) [14] cho rằng "Quá trình đặt thức ăn trực tuyến bao gồm việc khách hàng chọn nhà hàng mà họ muốn, xem xét thực đơn, chọn món và cuối cùng chọn để nhận hoặc giao hàng. Theo đó trang web (ứng dụng) thông báo cho khách hàng về chất lượng thực phẩm, thời gian chuẩn bị thực phẩm, thời gian thực phẩm sẵn sàng để nhận hoặc thời gian cần thiết để giao hàng."

Trong nghiên cứu này, nhóm tác giả thực hiện nghiên cứu ý định sử dụng ứng dụng giao thức ăn dựa trên nền tảng điện thoại thông minh. Cụ thể, việc đặt món ăn trực tuyến là quá trình đặt hàng thông qua ứng dụng di dộng giao thức ăn trực tuyến.

\section{2 Ý định}

Theo Ajzen (1991) [1] cho rằng "Ý định là một yếu tố tạo động lực, nó thúc đẩy một cá nhân sẵn sàng thực hiện hành vi". Đồng thời tác giả cũng chỉ ra rằng "Ý định bao gồm các yếu tố động cơ có ảnh hưởng đến hành vi của mỗi cá nhân; các yếu tố này cho thấy mức độ sẵn sàng hoặc nỗ lực mà mỗi cá nhân sẽ bỏ ra để thực hiện hành vi" 
Trong thuyết hành động hợp lý TRA (Theory of Reasoned Action) [10] được Ajzen \& Fishbein xây dựng từ cuối thập niên 60 của thế kỷ XX, được hiệu chỉnh mở rộng trong thập niên 70 đã chỉ ra rằng ý định là yếu tố quan trọng nhất để dự đoán hành vi tiêu dùng (Actual Behavior) và ý định bị ảnh hưởng bởi hai yếu tố: thái độ và chuẩn chủ quan. Bên cạnh đó mô hình chấp nhận công nghệ TAM (Technology Acceptance Model) được phát triển bởi Davis (1985) [7] dựa trên cơ sở của lý thuyết TRA cho thấy ý định được xem là tiền đề trực tiếp dẫn đến hành vi sử dụng công nghệ. Ngoài ra mối quan hệ giữa ý định và hành vi sử dụng công nghệ cũng được kiểm định bởi Joongho Ahn và ctg (2001) [16], Hasslinger và ctg (2007) [13] cùng với nghiên cứu của Hoàng Quốc Cường (2010) [6]. Chính vì thế trong nghiên cứu này, nhóm tác giả sẽ thực hiện các phân tích cũng như tập trung xem xét những yếu tố ảnh hưởng đến ý định sử dụng ứng dụng giao thức ăn của người tiêu dùng tại khu vực TP.Đà Nẵng.

\subsection{Mô hình và giả thuyết nghiên cứu}

Qua tham khảo các nghiên cứu về ý định sử dụng và chấp nhận công nghệ của các tác giả cũng như mô hình có liên quan, nghiên cứu đề xuất 6 nhân tố tác động đến ý định sử dụng ứng dụng giao thức ăn của người tiêu dùng tại $\mathrm{TP}$. Đà Nẵng. Trong đó nghiên cứu kế thừa các nhân tố từ 3 mô hình và 1 nghiên cứu: Nhân tố chuẩn chủ quan từ mô hình TRA (Ajzen \& Fishbein, 1975) [10]; Nhân tố nhận thức tính dễ sử dụng từ mô hình TAM (Davis, 1985) [7]; Nhân tố nhận thức rủi ro khi sử dụng từ mô hình E-CAM (Joongho Ahn và ctg, 2001) [16] và ba nhân tố mong đợi về giá, nhận thức sự thuận tiện và niềm tin từ nghiên cứu của Hasslinger và ctg (2007) [13].

(1) Nhận thức tính dễ sư dụg:

Theo Davis (1985) [7] thì "Nhận thức tính dễ sử dụng là cấp độ mà một người tin rằng sử dụng một ứng dụng đặc thù sẽ không cần nỗ lực". Các nghiên cứu của Joongho Ahn và ctg (2001) [16], Matthew K O Lee \& Christy M K Cheung (2005) [4], Nguyễn Duy Thanh và ctg (2015) [20], Lê Ngọc Đức (2008) [8], Hoàng Quốc Cường (2010) [6], Lee \& ctg (2017) [18] và Elango \& ctg (2018) [9] cũng chỉ ra rằng "Nhận thức tính dễ sử dụng" có tác động tích cực lên ý định sử dụng. Trong nghiên cứu này, nhận thức tính dễ sử dụng sẽ thể hiện ở chỗ người sử dụng ứng dụng giao thức ăn sẽ cảm thấy dễ dàng khi làm quen và dễ dàng thành thạo trong việc sử dụng ứng dụng đặt hàng để đáp ứng nhu cầu của họ khi cần. Do đó, tác giả đưa ra giả thuyết rằng:

Giả thuyết H1: Nhận thức tính dễ sử dụng có tác động tích cực (+) đến ý định sử dụng ứng dụng giao thức ăn của người tiêu dùng tại TP.Đà Nẵng

(2) Mong đợi về giá:

Hasslinger và ctg (2007) [13] đã đề cập đến việc khách hàng tin rằng mua hàng qua mạng sẽ giúp tiết kiệm thời gian, tiền bạc và ở họ có sự so sánh về giá. Và các nghiên cứu của Nguyễn Duy Thanh và ctg (2015) [20], Hoàng Quốc Cường (2010) [6] cũng chấp nhận rằng "Mong đợi về giá" có tác động tích cực lên ý định sử dụng. Trong nghiên cứu này, mong đợi về giá của khách hàng về ứng dụng giao thức ăn được thể hiện ở chồ khách hàng tin rằng việc sử dụng ứng dụng sẽ giúp họ tiết kiệm được tiền sản phẩm và giao dịch hơn so với dùng trực tiếp, thông qua các chương trình khuyến mãi. Do đó trong mô hình, tác giả đưa ra giả thuyết:

Giả thuyết H2: Mong đợi về giá có tác động tích cực (+) đến ý định sử dụng ứng dụng giao thức ăn của người tiêu dùng tại TP.Đà Nẵng

(3) Nhận thức sự thuận tiện:

Theo Hasslinger và ctg (2007) [13] đã đề cập đến việc người tiêu dùng nhận thấy rằng việc mua hàng qua mạng hay sử dụng dịch vụ giao nhận thức ăn trực tuyến giúp họ tiết kiệm được thời gian, công sức và có thể sử dụng dịch vụ bất cứ lúc nào khi có nhu cầu. Trong nghiên cứu này, nhân tố "Nhận thức sự thuận tiện" được tác giả nhìn nhận sẽ thể hiện qua việc ứng dụng giao thức ăn sẽ bổ trợ tích cực cho việc đặt hàng thức ăn của người tiêu dùng, giúp họ có thể đặt hàng với mức chi phí thấp, tiết kiệm thời gian. Do đó tác giả đề xuất giả thuyết $\mathrm{H} 3$ như sau:

Giả thuyết H3: Nhận thức sự thuận tiện có tác động tích cực (+) đến ý định sử dụng ứng dụng giao thức ăn của người tiêu dùng tại TP.Đà Nằng

(4) Nhận thức rủi ro khi sủ dụng:

Trong mô hình chấp nhận thương mại điện tử E-CAM (Joongho Ahn và ctg, 2001) [16] và thuyết nhận thức rủi ro TPR (Bauer, 1960) [3] cho rằng " Nhận thức rủi ro liên quan đến sản phẩm dịch vụ phản ánh sự lo lắng của người tiêu dùng về thông tin cá nhân khi sử dụng dịch vụ trực tuyến. Các rủi ro khi sử dụng có thể 
bao gồm: Lộ thông tin cá nhân, mất tài khoản, sản phẩm được nhận không đúng hoặc ảnh hưởng đến sức khỏe và vệ sinh an toàn thực phẩm". Trong nghiên cứu này, nhận thức rủi ro khi sử dụng sẽ thể hiện qua việc “ Người dùng ứng dụng trực tuyến càng nhận thức được rủi ro cao thì họ sẽ càng không sử dụng, hoặc không có ý định sử dụng". Từ đó, tác giả đưa ra giả thuyết rằng:

Giả thuyết H4: Nhận thức rủi ro khi sử dụng có tác động tiêu cực (-) đến ý định sử dụng ứng dụng giao thức ăn của người tiêu dùng tại TP.Đà Nẵng

(5) Niềm tin:

Nghiên cứu của Hasslinger và ctg (2007) [13] cho rằng niềm tin tác động tích cực đến ý định sử dụng của khách hàng. Đồng thời nhiều nghiên cứu cũng đã chấp nhận rằng niềm tin có vai trò lớn trong việc tác động đến ý định sử dụng của người tiêu dùng. Và theo nhận định của tác giả thì niềm tin của khách hàng thể hiện qua việc họ mong muốn sản phẩm nhận được phải đúng với những gì đã được mô tả. Do đó tác giả đề xuất giả thuyết:

Giả thuyết H5: Niềm tin có tác động tích cực (+) đến ý định sử dụng ứng dụng giao thức ăn của người tiêu dùng tại TP.Đà Nẵng

(6) Chuẩn chủ quan:

Theo Ajzen \& Fishbein (1975) [10] cho rằng "Chuẩn chủ quan hay ảnh hưởng từ xã hội là nhận thức của con người về áp lực xã hội để thể hiện hay không thể hiện hành vi. Các áp lực xã hội này xuất phát từ gia đình, bạn bè, đồng nghiệp, phương tiện truyền thông." Đồng thời kết quả nghiên cứu của Ajzen (1991) [1], Lê Ngọc Đức (2008) [8], Nguyễn Duy Thanh và ctg (2015) [20], Elango \& ctg (2018) [9] cũng chỉ ra rằng "Chuẩn chủ quan" có tác động tích cực lên ý định sử dụng. Do đó trong nghiên cứu này, tác giả nhìn nhận rằng, chuẩn chủ quan sẽ tác động lớn đến ý định sử dụng của khách hàng, hay nói cách khác tác động chuẩn chủ quan càng lớn thì khách hàng càng mong muốn sử dụng ứng dụng

Giả thuyết H6: Chuẩn chủ quan có tác động tích cực $(+)$ đến ý định sử dụng ứng dụng giao thức ăn của người tiêu dùng tại TP.Đà Nẵng

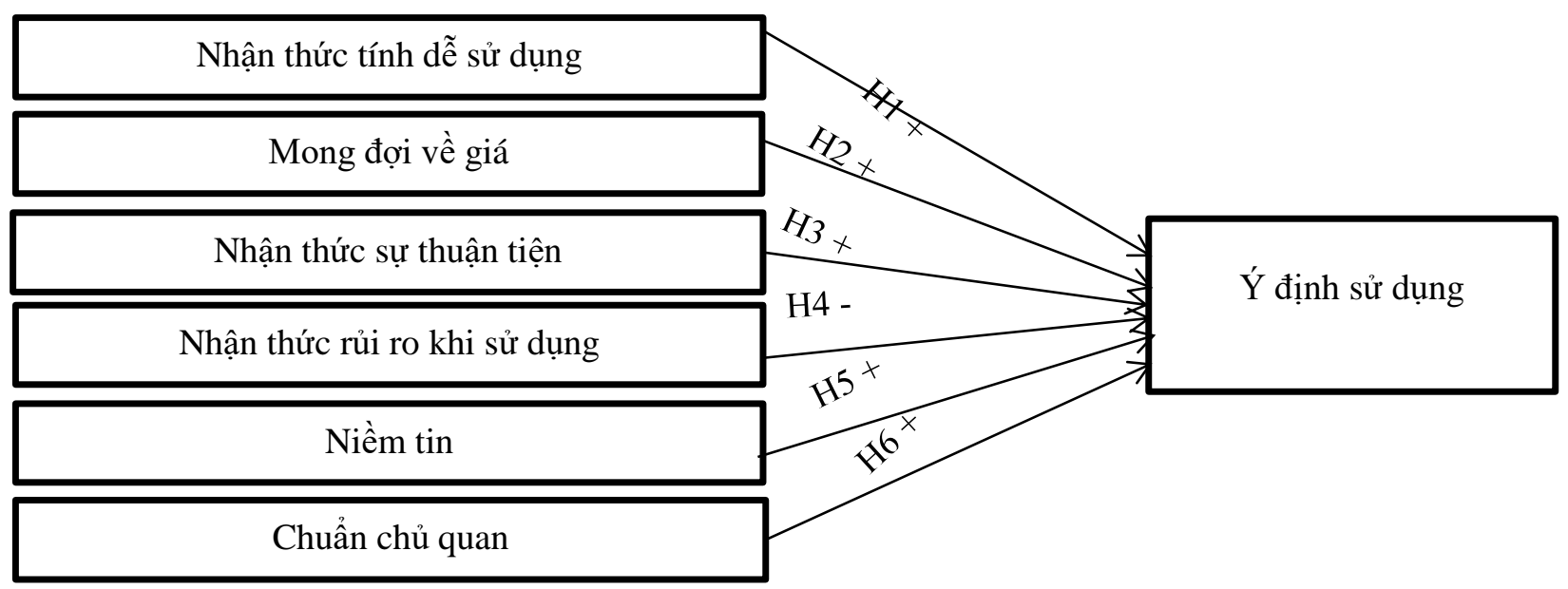

Hình 1. Mô hình đề xuất các yếu tố ảnh hưởng đến ý định sử dụng ứng dụng giao thức ăn của người tiêu dùng tại TP. Đà Nẵng

\section{PHƯƠNG PHÁP NGHIÊN CÚU}

Nguồn: Tác giả

Nghiên cứu được thực hiện thông qua hai phương pháp chính là nghiên cứu định tính và nghiên cứu định lượng. Nghiên cứu định tính được thực hiện thông qua hình thức phỏng vấn sơ bộ các cá nhân và chuyên gia bằng dàn bài phỏng vấn nhằm hiệu chỉnh thang đo. Nghiên cứu định lượng được tiến hành thông qua bảng câu hỏi khảo sát chính thức và tiến hành phân tích dữ liệu thu thập. Trong đó, đối tượng khảo sát là những người tiêu dùng đang sinh sống và làm việc tối thiểu 1 năm tại TP.Đà Nẵng với điều kiện những người tiêu dùng có sử dụng Internet và các thiết bị di động kết nối mạng không dây, đã từng biết đến ứng dụng giao thức ăn nhưng chưa từng sử dụng qua. Họ là những người tiêu dùng có nhu cầu đặt mua thực phẩm ngành hàng tiêu dùng nhanh và mong muốn sử dụng dịch vụ giao nhận tại nhà. Nhóm tác giả lựa 
chọn phương pháp lấy mẫu phi xác xuất, thuận tiện, cùng với việc tính toán kích thức mẫu dựa theo các tiêu chí sau: Kích cỡ mẫu phù hợp với kỹ thuật phân tích nhân tố EFA và tỉ lệ quan sát/biến đo lường là $5: 1$ $(5 \times 27=135)$ (Hair \& ctg, 2006) [12]. Trong nghiên cứu này, nhóm tác giả chọn kích thước mẫu là 350 nhằm đảm bảo độ tin cậy cao cũng như đề phòng các tác nhân làm giảm kích thước mẫu khi tiến hành khảo sát thực tế. Theo đó nghiên cứu đã tiến hành thu thập số liệu từ 350 người tiêu dùng, sau khi sàn lọc còn lại 326 trường hợp phù hợp để tiếp tục phân tích. Như vậy, số liệu được thu thập đảm bảo thực hiện tốt mô hình nghiên cứu, đồng thời để đo lường các khái niệm nghiên cứu trong mô hình, nhóm tác giả sử dụng thang đo Likert 5 mức độ từ 1 (Hoàn toàn không đồng ý) đến 5 (Hoàn toàn đồng ý). Dữ liệu sau khi được thu thập sẽ được xử lí và phân tích bằng phần mềm SPSS 20.0.

\section{KÊT QUẢ NGHIÊN CỨU}

\subsection{Tóm thông tin mẫu khảo sát}

Nghiên cứu đã tiến hành điều tra 350 người tiêu dùng, sau khi sàng lọc còn lại 326 phiếu khảo sát phù hợp để tiếp tục phân tích. Thống kê dữ liệu cho thấy phần lớn các đối tượng khảo sát thường có thời gian sử dụng Internet trung bình/ngày rơi vào khoảng trên 4 giờ chiếm phần lớn với 155 người trả lời $(47,5 \%$ trên tổng số đáp viên), chỉ có 8 người có thời gian sử dụng Internet dưới 30 phút/ ngày (chiếm $2,5 \%$ trên tổng số đáp viên). Nhóm tuổi chủ yếu của người khảo sát rơi vào khoảng từ 18 đến 35 tuổi chiếm tổng $69 \%$ trên số đáp viên. Trình độ học vấn Đại học-Cao Đẳng chiếm phần lớn với 199 người trả lời (chiếm 61,1\%), thấp nhất là số người có trình độ trên đại học với 19 người $(5,8 \%)$. Về nghề nghiệp, người tham gia khảo sát đang là nhân viên văn phòng chiếm đa số $(46,9 \%$ ), học sinh sinh viên có 97 người (chiếm $29,8 \%$ ) và nhóm cán bộ-Công viên chức có tỉ lệ đáp viên thấp nhất là $3,1 \%$. Tỉ lệ giữa nam và nữ trong cuộc khảo sát không có sự chênh lệch quá lớn (40,8\% đối với nam và $59,2 \%$ đối với nữ). Mức thu nhập của người tham gia khảo sát giao động từ 4 triệu trở lên với số người trả lời lần lượt là 99 người cho mức thu nhập từ 4 đến dưới 7 triệu $(30,4 \%)$ và 93 người cho mức thu nhập trên 7 triệu $(28,5 \%)$.

\subsection{Kết quả phân tích}

\subsubsection{Kiểm định độ tin cây thang đo với hệ số Cronbach's Alpha}

Kiểm định độ tin cậy thang đo (Cronbach's Alpha) được thực hiện đối với 7 nhân tố lần lượt như sau: Nhận thức tính dễ sử dụng, mong đợi về giá, nhận thức sự thuận tiện, nhận thức rủi ro khi sử dụng, niềm tin, chuẩn chủ quan và ý định sử dụng. Kết quả kiểm định Cronbach's Alpha cho thấy rằng tất cả các biến quan sát trong mỗi nhân tố đều có hệ số tương quan biến tổng lớn hơn 0,3 và 7 nhân tố của mô hình đều có hệ số Cronbach's Alpha lớn hơn 0,6 (Theo Nunnally \& Bernstein, 1994) [19]. Do đó có thể kết luận rằng tất cả các biến quan sát trong mỗi nhóm nhân tố đều đủ độ tin cậy để thực hiện các bước tiếp theo (xem bảng 1).

Bảng 1. Kết quả kiểm định Cronbach's Alpha

\begin{tabular}{|l|c|c|c|}
\hline \multicolumn{1}{|c|}{ Thang đo } & $\begin{array}{c}\text { Tổng } \\
\text { sốn } \\
\text { biến }\end{array}$ & Biến thành phần & $\begin{array}{c}\text { Hệ số } \\
\text { Cronbach's } \\
\text { Alpha }\end{array}$ \\
\hline Nhận thức tính dễ sử dụng & 4 & DDSD1, DDSD2, DDSD3, DDSD4. & 0,694 \\
\hline Mong đợi về giá & 4 & MDVG1, MDVG2, MDVG3, MDVG4. & 0,771 \\
\hline Nhận thức sự thuận tiện & 4 & TT1, TT2, TT3, TT4. & 0,818 \\
\hline Nhận thức rủi ro khi sử dụng & 4 & RR1, RR2, RR3, RR4. & 0,807 \\
\hline Niềm tin & 3 & NT1, NT2, NT3 & 0,803 \\
\hline Chuẩn chủ quan & 4 & CCQ1, CCQ2, CCQ3, CCQ4 & 0,767 \\
\hline Ý định sử dụng & 4 & YDSD1, YDSD2, YDSD3, YDSD4 & 0,881 \\
\hline
\end{tabular}

4.2.2 Phân tích nhân tố khám phá-EFA

Nguồn: Kết quả xư lý

Sau khi thực hiện kiểm định độ tin cậy thang đo với hệ số Cronbach's Alpha, tất cả các biến độc lập (23 biến) tiếp tục được đưa vào để phân tích nhân tố khám phá EFA. Kết quả sau lần phân tích nhân tố khám phá EFA đầu tiên thì có 1 biến quan sát bị loại là NT3 vì có hệ số tải nhân tố $=0,465<0,5$ ( Hair \& ctg, 1998, 2006) [11] [12]. Nhóm tác giả tiến hành loại biến quan sát NT3 và thực hiện phân tích lần thứ hai với các biến độc lập còn lại (22 biến). Kết quả sau lần phân tích thứ hai được trình bày và tóm lượt thông qua bảng 2 . 
Hệ số KMO (Kaiser - Meyer - Olkin) là 0,813 thỏa mãn yêu cầu của phân tích nhân tố $0,5 \leq \mathrm{KMO} \leq 1$ (Trọng Ngọc Hoàng \& Nguyễn Mộng Chu, 2008) [15] nên kết luận phân tích nhân tố là phù hợp với dữ liệu thực tế. Hệ số Sig. $=0,000<0,05$ nên kết luận các biến quan sát tương quan với nhau trong tổng thể, có ý nghĩa thống kê. Chỉ số dừng nhân tố (Eigenvalue) là $1,037>1$ và tồng phương sai trích (Variance Extracted) là $67,759 \%$ điều đó cho thấy $67,759 \%$ sự biến thiên của dữ liệu được giải thích bởi 6 nhân tố trong mô hình (Hair và ctg, 1998, 2006) [11][12]. Kết quả phân tích nhân tố khám phá của các biến độc lập cho thấy có 6 nhân tố được rút ra và các nhân tố này không có sự xáo trộn, nên tên biến được giữ nguyên (DDSD, MDVG, TT, RR, NT, CCQ). Hệ số tải nhân tố của 22 biến quan sát còn lại đều $>0,5$ do đó các biến này có độ tin cậy và thỏa điều kiện khi phân tích nhân tố

Tương tự, phân tích nhân tố khám phá EFA cho biến phụ thuộc được tiến hành và thu được kết quả: Hệ số Kaiser - Meyer - Olkin $(\mathrm{KMO})=0,831$ thỏa mãn yêu cầu của phân tích nhân tố $0,5 \leq \mathrm{KMO} \leq 1$; Giá trị Sig Bartlett's Test $=0,000<0,05$; Chỉ số dừng nhân tố (Eigenvalue) $=2,955>1$; Tổng phương sai trích(Variance Extracted $)=73,882 \%>50 \%$ và hệ số tải nhân tố của các biến quan sát đều $>0,5$ nên thỏa yêu cầu.

Bảng 2. Kết quả phân tích nhân tố khám phá EFA cho các biến độc lập

\begin{tabular}{|c|c|c|c|c|c|c|}
\hline \multicolumn{7}{|c|}{ MA TRẬN XOAY NHÂN TỐ } \\
\hline TÊNN BIẾN & \multicolumn{6}{|c|}{ NHÂN TỐ } \\
\hline MÃ HÓA & 1 & 2 & 3 & 4 & 5 & 6 \\
\hline TT3 & 0,783 & & & & & \\
\hline TT1 & 0,759 & & & & & \\
\hline TT4 & 0,742 & & & & & \\
\hline TT2 & 0,633 & & & & & \\
\hline RR4 & & 0,836 & & & & \\
\hline RR1 & & 0,817 & & & & \\
\hline RR3 & & 0,782 & & & & \\
\hline RR2 & & 0,604 & & & & \\
\hline MDVG2 & & & 0,772 & & & \\
\hline MDVG1 & & & 0,720 & & & \\
\hline MDVG4 & & & 0,668 & & & \\
\hline MDVG3 & & & 0,532 & & & \\
\hline $\mathrm{CCQ} 2$ & & & & 0,850 & & \\
\hline CCQ3 & & & & 0,781 & & \\
\hline CCQ1 & & & & 0,661 & & \\
\hline CCQ4 & & & & 0,635 & & \\
\hline DDSD2 & & & & & 0,753 & \\
\hline DDSD3 & & & & & 0,656 & \\
\hline DDSD1 & & & & & 0,623 & \\
\hline DDSD4 & & & & & 0,601 & \\
\hline NT1 & & & & & & 0,705 \\
\hline NT2 & & & & & & 0,703 \\
\hline Hệ số KMO & & & & 0,813 & & \\
\hline Giá trị Sig Bartlett's Test & & & & 0,000 & & \\
\hline $\begin{array}{l}\text { Chỉ số dừng nhân tố } \\
\text { (Eigenvalue) }\end{array}$ & & & & $1, \mathbf{0 3 7}$ & & \\
\hline Tổng phương sai trích & & & & $7,759 \%$ & & \\
\hline
\end{tabular}

Nguồn: Kết quả xử lý 


\subsubsection{Phân tích hồi quy đa biến}

*Phân tích tuơng quan Pearson

Bảng 3. Kết quả phân tích tương quan Pearson

\begin{tabular}{|l|c|c|c|c|c|c|c|}
\hline & YDSD & DDSD & MDVG & TT & RR & NT & CCQ \\
\hline YDSD & 1 & & & & & & \\
\hline DDSD & $0,479^{* *}$ & 1 & & & & & \\
\hline MDVG & $0,558^{* *}$ & $0,516^{* *}$ & 1 & & & & \\
\hline TT & $0,549^{* *}$ & $0,434^{* *}$ & $0,549^{* *}$ & 1 & & & \\
\hline RR & 0,055 & $-0,037$ & 0,068 & $-0,004$ & 1 & & \\
\hline NT & $0,565^{* *}$ & $0,378^{* *}$ & $0,406^{* *}$ & $0,537^{* *}$ & $-0,318^{* *}$ & 1 & \\
\hline CCQ & $0,479^{* *}$ & $0,221^{* *}$ & $0,433^{* *}$ & $0,267^{* *}$ & $0,143^{* *}$ & $0,269^{* *}$ & 1 \\
\hline
\end{tabular}

**. Tương quan ở mức ý nghĩa 1\% (kiểm định 2 phía).

Nguồn: Kết quả xử lý

Trước khi thực hiện phân tích hồi quy đa biến, tác giả thực hiện phân tích tương quan Pearson để kiểm định tương quan giữa biến phụ thuộc là ý định sử dụng (YDSD) và các biến độc lập, đồng thời cũng kiểm định độ tương quan giữa các biến độc lập với nhau. Từ kết quả phân tích tương quan Pearson cho thấy các nhân tố đều có mối quan hệ chặt chẽ với nhân tố ý định sử dụng ở mức ý nghĩa $1 \%$. Cụ thể, các nhân tố DDSD, MDVG, TT, NT, CCQ có mối tương quan cùng chiều với nhân tố ý định sử dụng (YDSD). Nhân tố RR có mối tương quan ngược chiều với nhân tố ý định sử dụng (YDSD)

*Phân tích tuoong quan hồi quy

Bảng 4. Kết quả phân tích hồi quy

\begin{tabular}{|c|c|c|c|c|c|c|c|}
\hline \multirow[t]{2}{*}{$\begin{array}{c}\text { Nhân } \\
\text { tố }\end{array}$} & \multicolumn{2}{|c|}{$\begin{array}{c}\text { Hệ số hồi quy chưa } \\
\text { chuẩn hóa }\end{array}$} & \multirow{2}{*}{$\begin{array}{c}\begin{array}{c}\text { Hệ số hồi quy } \\
\text { chuẩn hóa }\end{array} \\
\text { Beta }\end{array}$} & \multirow[t]{2}{*}{$\mathbf{t}$} & \multirow[t]{2}{*}{ Sig. } & \multicolumn{2}{|c|}{ Collinearity Statistics } \\
\hline & $\mathbf{B}$ & Sai số chuẩn & & & & Tolerance & VIF \\
\hline DDSD & 0,169 & 0,048 & 0,161 & 3,510 & 0,001 & 0,686 & 1,458 \\
\hline MDVG & 0,149 & 0,050 & 0,154 & 2,956 & 0,003 & 0,530 & 1,888 \\
\hline TT & 0,179 & 0,051 & 0,179 & 3,532 & 0,000 & 0,560 & 1,784 \\
\hline $\mathrm{RR}$ & $-0,007$ & 0,036 & $-0,008$ & $-0,195$ & 0,845 & 0,810 & 1,235 \\
\hline NT & 0,244 & 0,045 & 0,274 & 5,387 & 0,000 & 0,560 & 1,786 \\
\hline $\mathrm{CCQ}$ & 0,243 & 0,041 & 0,257 & 5,944 & 0,000 & 0,774 & 1,293 \\
\hline \multicolumn{8}{|c|}{ Giá trị R2 hiệu chỉnh $=0,531$} \\
\hline \multicolumn{8}{|c|}{ SigF $=0,000$} \\
\hline
\end{tabular}

Nguồn: Kết quả xủ ly

Kết quả phân tích tương quan hồi quy của các nhân tố trong mô hình như sau: Giá trị R2 hiệu chỉnh là 0,531 (hay 53,1\%), Sig $=0,000 \leq 0,05$ có nghĩa tồn tại mô hình hồi quy tuyến tính giữa ý định sử dụng và 6 nhân tố trong mô hình. Kiểm định $\mathrm{F}$ có Sig. $=0,000(<0,05)$ rất nhỏ cho thấy mô hình sử dụng là phù hợp với dữ liệu thực tế. Hệ số Durbin-Watson $(\mathrm{d})=1,790$ nằm trong khoảng $(\mathrm{du}=1,725$; 4-du $=2,275)$ nên kết luận không có hiện tượng tự tương quan giữa các phần dư trong mô hình, mô hình có ý nghĩa thống kê; hệ số phóng đại phương sai VIF của các biến trong mô hình đều rất nhỏ, có giá trị từ 1,235 đển 1,888 nhỏ hơn 10 thỏa điều kiện VIF < 10 kết luận không vi phạm giả thuyết hiện tượng đa cộng tuyến, mô hình có ý nghĩa thống kê.

Bảng 5. Tóm tắt kết quả kiểm định giả thuyết

\begin{tabular}{|c|c|c|c|c|}
\hline $\begin{array}{c}\text { GIẢ } \\
\text { THUYẾT }\end{array}$ & NỘI DUNG & $\begin{array}{l}\text { HỆ SỐ BETA } \\
\text { CHUẤN HÓA }\end{array}$ & SIG & $\begin{array}{l}\text { KẾT } \\
\text { QUẢ }\end{array}$ \\
\hline H1 & $\begin{array}{l}\text { Nhận thức tính dễ sử dụng có tác động tích } \\
\text { cực }(+) \text { đến ý định sử dụng }\end{array}$ & 0,161 & 0,001 & $\begin{array}{l}\text { Chấp } \\
\text { nhận }\end{array}$ \\
\hline H2 & $\begin{array}{l}\text { Mong đợi về giá có tác động tích cực }(+) \text { đến } \\
\text { ý định sử dụng }\end{array}$ & 0,154 & 0,003 & $\begin{array}{l}\text { Chấp } \\
\text { nhận }\end{array}$ \\
\hline
\end{tabular}



CỦA NGƯỜI TIÊU DÙNG TẠI THÀNH PHỐ ĐÀ NĂNG

\begin{tabular}{|l|l|c|c|c|}
\hline $\mathbf{H 3}$ & $\begin{array}{l}\text { Nhận thức sự thuận tiện có tác động tích cực } \\
(+) \text { đến ý định sử dụng }\end{array}$ & 0,179 & 0,000 & $\begin{array}{c}\text { Chấp } \\
\text { nhận }\end{array}$ \\
\hline $\mathbf{H 4}$ & $\begin{array}{l}\text { Nhận thức rủi ro khi sử dụng có tác động tiêu } \\
\text { cực (-) đến ý định sử dụng }\end{array}$ & $-0,008$ & $\begin{array}{c}\text { Chừ đủ } \\
\text { bằng } \\
\text { chứng } \\
\text { để kêt } \\
\text { luận }\end{array}$ \\
\hline $\mathbf{H 5}$ & $\begin{array}{l}\text { Niềm tin có tác động tích cực }(+) \text { đến ý định } \\
\text { sử dụng }\end{array}$ & 0,274 & 0,000 & $\begin{array}{c}\text { Chấp } \\
\text { nhận }\end{array}$ \\
\hline $\mathbf{H 6}$ & $\begin{array}{l}\text { Chuẩn chủ quan có tác động tích cực }(+) \text { đến } \\
\text { ý định sử dụng dịch }\end{array}$ & 0,257 & 0,000 & $\begin{array}{c}\text { Chấp } \\
\text { nhận }\end{array}$ \\
\hline
\end{tabular}

Nguồn: Kết quả xủ lý

Kết quả sau phân tích có 5 nhân tố ảnh hưởng đến ý định sử dụng gồm: Nhận thức tính dễ sử dụng (DDSD), mong đợi về giá (MDVG), nhận thức sự thuận tiện (TT), niềm tin (NT) và chuẩn chủ quan (CCQ) với sig đều $<0,05$. Nhân tố nhận thức rủi ro khi sử dụng $(\mathrm{RR})$ chưa đủ bằng chứng để kết luận có tác động trong trường hợp phạm vi của đề tài vì sig $=0,845>0,05$. Thực tế cho thấy, dịch vụ giao nhận thức ăn trực tuyến qua ứng dụng trên điện thoại thường sử dụng hình thức thanh toán trực tiếp bằng tiền mặt hoặc thanh toán qua thẻ ngân hàng nhưng có sự xác nhận rõ ràng giữa người tiêu dùng và phía cung cấp dịch vụ thông qua ứng dụng, vì thế khách hàng sẽ dễ dàng kiểm soát được quá trình giao nhận mà không cảm thấy quá lo lắng về rủi ro tài chính hoặc các rủi ro liên quan đến thông tin cá nhân cơ bản có thể xảy ra. Bên cạnh đó, mặt hàng giao dịch qua ứng dụng giao nhận thức ăn thuộc dạng hàng tiêu dùng nhanh, giá trị lại không quá cao. Với kết quả kiểm định giả thuyết được trình bày tại bảng 5 và kết quả thu thập trong phạm vi nghiêm cứu của đề tài, thì phương trình hồi quy chuẩn hóa thể hiện các yếu tố ảnh hưởng đến ý định sử dụng ứng dụng giao thức ăn của người tiêu dùng tại TP.Đà Nẵng như sau:

YDSD=0,161*DDSD +0,154*MDVG + 0,179*TT + 0,274*NT + 0,257*CCQ (1)

Qua bảng trên ta thây được mức độ ảnh hưởng của các yếu tố đối với ý định sử dụng ứng dụng giao thức ăn của người tiêu dùng tại TP.Đà Nẵng. Kết quả của nghiên cứu khá tương đồng với những nghiên cứu trước đó và phù hợp với thực tế thị trường. Trong đó, yếu tố Niềm tin $(\beta=0,274)$ tác động mạnh nhất đến ý định sự dụng. Thực tế cho thấy, khách hàng thường xuyên đánh giá cao niềm tin khi có ý định sử dụng ứng dụng giao thức ăn. Họ có xu hướng lựa chọn những thương hiệu uy tín, mức độ bảo mật thông tin cao, chất lượng sản phẩm đạt yêu cầu để sử dụng vì hiện nay đang có quá nhiều dịch vụ thương mại điện tử nhưng mức độ đảm bảo các thông tin cho khách hàng lại bị hạn chế khiến nhiều người mất lòng tin với doanh nghiệp. Do đó niềm tin về ứng dụng, dịch vụ càng cao thì càng thúc đẩy ý định sử dụng của người dùng. Yếu tố có tác động mạnh thứ hai là Chuẩn chủ quan $(\beta=0,257)$. Trong nghiên cứu này cũng cho thấy rằng ảnh hưởng từ phương tiện truyền thông tác động mạnh đến người sử dụng. Điều này là phù hợp vì ngày càng có nhiều người sử dụng mạng xã hội và tiếp cận nhiều với phương tiện truyền thông hơn. Mức độ tác động (ảnh hưởng) càng cao thì người tiêu dùng sẽ dễ dàng có ý định sử dụng.

Kết tiếp, nhận thức sự thuận tiện $(\beta=0,179)$ và Nhận thức tính dễ sử dụng $(\beta=0,161)$ cũng có ảnh hưởng đến ý định sử dụng. Điều này hoàn toàn tương thích với nhận định tổng quan và các cuộc khảo sát trước đó khi cho rằng khách hàng sẽ thường xuyên đánh giá cao các ứng dụng có hệ thống thông tin đơn giản và dễ tiếp cận hơn, thao tác đơn giản và tính năng rỏ ràng, dễ hiểu... Đồng nghĩa là thông tin, các thao tác cũng như thời gian đặt hàng càng đơn giản, thuận tiện thì ý định sử dụng ứng dụng sẽ tăng lên.

Sau cùng, yếu tố Mong đợi về giá $(\beta=0,154)$ có ảnh hường đến ý định sử dụng. Có thể dễ dàng hiểu được kết quả này vì đối với ứng dụng giao thức ăn thì khách hàng không mấy đề cao vấn đề về giá cả sản phẩm do tính chất tương đồng giá giữa các thương hiệu, thay vào đó họ quan tâm nhiều về các chương trình hậu mãi hơn là giá niêm yết của sản phẩm trực tuyến. Vậy nên yếu tố này chỉ ảnh hưởng đến ý định với mức độ thấp.

\section{KẾT LUẬN VÀ HÀM Ý QUẢN TR!}

\subsection{Kết luận}

Có thể nhận thấy được rằng lĩnh vực dịch vụ thương mại trực tuyến nói chung và ứng dụng giao thức ăn nói riêng đang ngày càng phát triển mạnh trong thời đại công nghệ đang lên ngôi như hiện nay. Đặc biệt là thị trường Việt Nam đang có tiềm năng phát triển rất lớn trong lĩnh vực này khi số lượng các Công ty gia 
tăng không ngừng và ngày càng phủ sóng khắp cả nước. Kết quả nghiên cứu đã tìm ra các yếu tố ảnh hưởng đến ý định sử dụng ứng dụng giao thức ăn của người tiêu dùng tại Đà Nẵng, đặc biệt là hai yếu tố niềm tin và chuẩn chủ quan. Hai yếu tố này được nhận định là có ảnh hưởng trực tiếp và cùng chiều đến ý định sử dụng và hơn thế nữa, hai nhân tố này có ảnh hưởng mạnh hơn cả ba nhân tố truyền thống trong mô hình TRA và mô hình TAM (là nhận thức sự hữu ích, nhận thức sự tín nhiệm và nhận thức tính dễ sử dụng). Các nhà nghiên cứu trong lĩnh vực này có thể xem đây như một mô hình tham khảo trong việc phát triển các hướng nghiên cứu tiểp theo.

Nghiên cứu đã đóng góp thêm tài liệu khoa học cho lĩnh vực giao nhận thức ăn trực tuyến đang còn khá mới mẻ cũng như thông qua mô hình và kết quả nghiên cứu có thể giúp xác định được các yếu tố tác động đến ý đinh sử dụng ứng dụng giao thức ăn của người tiêu dùng tại Đà Nẵng. Bằng việc xây dựng mô hình nghiên cứu sử dụng các lý thuyết nền từ 3 mô hình: mô hình TRA (Ajzen \& Fishbein, 1975) [10], TAM (Davis, 1985) [7] và E-CAM (Joongho Ahn và ctg, 2001) [16] và nghiên cứu của Hasslinger và ctg (2007) [13] để làm nền tảng, tác giả đã cung cấp một cái nhìn đầy đủ hơn về vấn đề nghiên cứu.

Ngoài việc xác định các yếu tố ảnh hưởng đến ý định sử dụng, nghiên cứu còn giúp các doanh nghiệp về cung cấp và phát triển ứng dụng giao thức ăn trực tuyến nhìn nhận được vấn đề mà giao nhận trực tuyến cần quan tâm để cải thiện được dịch vụ cũng như thu hút được thêm khách hàng cho doanh nghiệp mình, đồng thời hỗ trợ nhà quản lý của các doanh nghiệp về ứng dụng giao thức ăn đưa ra được những phương án nâng cao sự quan tâm của khách hàng, đưa ra được những giải pháp thúc đẩy ý định sử dụng của người tiêu dùng, đổi mới để cạnh tranh lại với các đối thủ đang ngày càng lớn mạnh ... nhằm góp phần nâng cao hiệu quả kinh doanh cho doanh nghiệp.

\subsection{Hàm ý quản trị}

Theo kết quả nghiên cứu thì có 5 nhân tố tác động đến ý định sử dụng ứng dụng giao thức ăn của người tiêu dùng tại TP. Đà Nẵng: Niềm tin, Chuẩn chủ quan, Nhận thức sự thuận tiện, Nhận thức tính dễ sử dụng và Mong đợi về giá. Tùy thuộc vào mức độ ảnh hưởng của từng yếu tố, nhóm tác giả sẽ đề xuất một số kiến nghị nhằm gợi ý cho các doanh nghiệp có thể cải thiện cũng như đưa ra được các phương án tiếp thị hiệu quả hơn.

Niềm tin: Yếu tố niềm tin có tác động mạnh nhất đến ý định sử dụng của người tiêu dùng. Khách hàng sẽ có ý định sử dụng dịch vụ khi họ thỏa mãn được các yếu tố về niềm tin: giá cả, hình thức giao nhận, thông tin....Do đó để nâng cao ý định sử dụng ứng dụng giao thức ăn của người tiêu dùng tại TP. Đà Nẵng thì nhóm tác giả đưa ra một số kiến nghị sau: Thường xuyên cập nhập thông tin về ứng dụng, hình thức giao nhận, hình thức thanh toán thường xuyên và chính xác để giúp khách hàng có thể dễ dàng xem xét và sử dụng; Cập nhập thông tin đơn hàng cụ thể chi tiết cũng như thông tin về nhân viên giao nhận để khách hàng dễ dàng liên lạc khi cần thiết...; Các chương trình khuyến mãi cần rỏ ràng và được áp dụng đúng với những gì đã thông báo cho khách hàng, tránh tình trạng chương trình thực hiện không phù hợp sẽ làm khách hàng khó chịu; Bảo mật thông tin cá nhân của khách hàng, đảm bảo an toàn hàng hóa trong quá trình vận chuyển, tránh hư hỏng và thiếu hàng hóa của khách.

Chuẩn chủ quan: Các đối tượng ảnh hưởng đến khách hàng về việc sử dụng dịch vụ giao nhận thức ăn trực tuyến gồm có gia đình, người thân, bạn bè và các phương tiện truyền thông. Theo kết quả khảo sát thì mức độ tác động của các phương tiện truyền thông như facebook, youtube, instagram... chiếm phần lớn với điểm trung bình khá cao. Điểu này có thể cho thấy được rằng, người dùng có xu hướng tiếp cận và sử dụng ứng dụng giao thức ăn thông qua các phương tiện truyền thông nhiều hơn các hình thức tiếp cận khác. Do đó nhóm tác giả đề xuất một số kiến nghị về vấn đề này như sau: Các công ty nên thường xuyên đẩy mạnh các chương trình quảng cáo, truyền thông về ứng dụng trên các diễn đàng hoặc mạng xã hội như Facebook, Youtube...; Đưa ra nhiều chương trình khuyến mãi hấp dẫn trên các phương tiện truyền thông để giúp tiếp cận và thu hút nhiều lượt khách hàng sử dụng ứng dụng hơn; Thường xuyên có các phần quà thưởng cho các thành viên giới thiệu ứng dụng đến nhiều người bằng các vouchers giảm giá hoặc đổi các phần quà có giá trị khác; Tạo fanpage và thực hiện tương tác với khách hàng thông qua các trò chơi nhận thưởng với phần quà là các mã giảm giá khi đặt hàng để kích thích khách hàng tham gia và sử dụng ứng dụng

Nhận thức sự thuận tiện và Nhận thức tính dễ sử dụng: Đây là hai yếu tố tác động trung bình đển ý định sử dụng. Người dùng mặc dù đánh giá khá cao về sự thuận tiện khi sử dụng ứng dụng cũng như các ứng dụng hiện nay đều đa phần có bố cục rỏ ràng, dễ sử dụng đối với người tiêu dùng, tuy nhiên sự thay đổi và cập nhập thường xuyên cũng như một số hạn chế về hình thức đặt hàng trên ứng dụng cũng khiến người dùng 


\section{NGHIÊN CÚU CÁC YÊU TỐ ẢNH HƯỞNG ĐÊN Ý ĐỊNH SỬ DỰG UÚNG DỤNG GIAO THỨC ĂN CỦA NGƯÒ̀I TIÊU DÙNG TẠI THÀNH PHỐ ĐÀ NÃNG}

không đánh giá cao. Một số kiến nghị cho vấn đề này được tác giả đưa ra như sau: Các doanh nghiệp cần cải thiện quy trình đặt hàng sao cho thuận tiện hơn cho khách hàng, các thông tin về sản phẩm cần rỏ ràng, mức giá cũng cần thống nhất hơn để khách hàng thuận tiện trong việc đặt hàng; Thông tin về địa chỉ giao nhận hoặc địa chỉ quán cần độ chính xác cao, mức phí giao nhận cũng cần có thông tin cụ thể, tránh việc thay đổi đột ngột khiến khách hàng khó quản lý; Đơn giản hóa hình thức đặt hàng để người dùng dễ dàng thao tác.

Mong đợi về giá: Đây là nhân tố có mức tác động thấp nhất đối với ý định sử dụng của người tiêu dùng. Trên thực tế việc đặt hàng thức ăn thông qua ứng dụng đều đã được định giá sẵn, không có sự chênh lệch giá giữa các quán ăn của các thương hiệu. Người tiêu dùng thay vào đó sẽ quan tâm nhiều tới khuyến mãi và phí giao nhận hơn là mức giá sản phẩm. Do đó, nhóm tác giả cũng kiến nghị một số ý kiến sau: Cần có thêm các chương trình khuyến mãi giảm giá để kích thích nhu cầu của người sử dụng; Thường xuyên công khai truyền thông các chương trình mới giúp tăng mức độ sử dụng của người tiêu dùng; Giảm mức phí giao nhận cho người sử dụng khi họ đặt hàng thường xuyên.

\subsection{Hạn chế và hướng nghiền cứu tiếp theo}

Tuy rằng bài nghiên cứu đã đáp ứng được hết các mục tiêu đã đề ra tuy nhiên thì trong quá trình thực hiện, bài nghiên cứu cũng gặp một số những hạn chế nhất định:

- Thời gian thực hiện nghiên cứu hạn chế cũng như nguồn lực không đủ để tiếp cận được nhiều khách hàng nên kích thước mẫu nghiên cứu vẫn chưa đủ lớn. Do đó trong nghiên cứu tiếp theo có thể thực hiện với kích thước mẫu lớn hơn và phương pháp chọn mẫu có tính đại diện cao hơn để giúp xác định đúng đối tượng cho nghiên cứu.

- Nghiên cứu chỉ xét phương diện các yếu tố ảnh hưởng đến ý định sử dụng mà chưa đi sâu vào tâm lý cũng như quyết định mua của khách hàng. Do đó các nghiên cứu sau cần xem xét thêm mối quan hệ giữa ý định sử dụng và hành vi tiêu dùng.

\section{TÀI LIÊU THAM KHẢO}

[1] Ajzen, I. (1991). The theory of planned behavior. Organizational behavior and human decision processes, 50(2), $179-211$.

[2] Ban Chấp hành Đảng bộ TP. Đà Nẵng. (2018). Báo cáo Hội nghị lần thư 15. Đà Nẵng, Việt Nam.

[3] Bauer, R. A. (1960). Consumer behavior as risk taking. Chicago, IL, 384-398.

[4] Cheung, C. M., \& Lee, M. K. (2005). Research framework for consumer satisfaction with internet shopping.

[5] Công ty Cổ phần Nghiên cứu Thị trường GCOMM. (2018). Nghiên cứu về mức độ hài lòng của nguoòi dùng đối với các dịch vu đặt món trục tuyến tại thị truoơng Việt Nam.

[6] Cường, H. Q. (2010). Nghiên cứu các yếu tố ảnh hưởng đến ý định sủ dụng dịch vu mua hàng điện tử qua mạng (Doctoral dissertation, Luận văn Thạc sĩ-Quản trị kinh doanh-Trường Đại học Bách Khoa TP. HCM).

[7] Davis, N. (1985). The last two years of Salvador Allende (p. 72). Ithaca, NY: Cornell University Press.

[8] Đức, L. N. (2008). Khảo sát một số yếu tố tác động đến xu huớng sư dụng thanh toán điện tủ. Luận văn Thạc Sĩ Quản trị Kinh Doanh, Đại học Bách Khoa, TPHCM.

[9] Elango, D., Dowpiset, K., \& Chantawaranurak, J. (2018). A Study on Factors Impacting Consumers' Intention to Use On-demand Food Delivery Applications in Bangkok, Thailand. Thailand (August 24, 2018).

[10] Fishbein, M., \& Ajzen, I. (1975). Belief, attitude, and behavior: An introduction to theory and research. Reading, Mass.: Addison Wessley.

[11] Hair, J. F., Anderson, R. E., Tatham, R. L., \& Black,W. C. (1998), Multivariate data analysis (5 ${ }^{\text {th }}$ ed). Upper Saddle River, NJ: Prentice Hall.

[12] Hair, J. F., Black, W. C., Babin, B. J., Anderson, R. E., \& Tatham, R. L. (2006). Multivariate data analysis (6 $6^{\text {th }}$ ed). Upper Saddle River, NJ: Prentice Hall. 


\section{NGHIÊN CÚUU CÁC YÊU TỐ ẢNH HƯỞNG ĐÊN Ý ĐỊNH SỬ DƯNG ỨNG DỤNG GIAO THỨC ĂN CỦA NGƯÒ̀I TIÊU DÙNG TẠI THÀNH PHỐ ĐÀ NÃNG}

[13] Hasslinger, A. S. H. (2007). Customer behaviour in Online Shopping. Kristianstad University, Master thesis.

[14] He, Z., Han, G., Cheng, T. C. E., Fan, B., \& Dong, J. (2018). Evolutionary food quality and location strategies for restaurants in competitive online-to-offline food ordering and delivery markets: An agent-based approach. International Journal of Production Economics.

[15] Hoàng, T., \& Chu, N. M. N. (2008). Phân tích dữ liệu nghiên cứu với SPSS-tập 1.

[16] JoonghoAhn, J. P. (2001). Risk Focused e-Commerce adoption model- A cross Country Study. Carlson School of Management. University of Minnesota.

[17] Kimes, S. E., \& Laqué, P. F. (2011). Online, Mobile, and Text Food Ordering in the US Restaurant Industry. Cornell Hospitality Report, 11(7), 6-15.

[18] Lee, E. Y., Lee, S. B., \& Jeon, Y. J. J. (2017). Factors influencing the behavioral intention to use food delivery apps. Social Behavior and Personality: an international journal, 45(9), 1461-1473.

[19] Nunnally, J. C., \& Bernstein, I. H. (1994). Psychological theory. New York, NY: MacGraw-Hill, 131-147.

[20] Thanh, N. D., Châu, H. T. M., \& Tuân, N. M. (2015). Chấp nhận và sử dụng công nghệ: Một nghiên cứu về dịch vụ taxi uber. Tạp chí Phát triển Khoa học và Công nghệ, 18(4Q), 84-93.

Ngày nhận bài: 12/09/2019

Ngày chấp nhận đăng: 25/12/2019 